\title{
Publisher correction: Young inversion with multiple linked QTLs under selection in a hybrid zone
}

Cheng-Ruei Lee, Baosheng Wang, Julius P. Mojica, Terezie Mandáková, Kasavajhala V. S. K. Prasad, Jose Luis Goicoechea, Nadeesha Perera, Uffe Hellsten, Hope N. Hundley, Jenifer Johnson, Jane Grimwood, Kerrie Barry, Stephen Fairclough, Jerry W. Jenkins, Yeisoo Yu, Dave Kudrna, Jianwei Zhang, Jayson Talag, Wolfgang Golser, Kathryn Ghattas, M. Eric Schranz, Rod Wing, Martin A. Lysak, Jeremy Schmutz, Daniel S. Rokhsar and Thomas Mitchell-Olds

Nature Ecology \& Evolution 1, 0119 (2017); published online 3 April 2017; corrected online 24 April $2017 ; 25$ August 2017.

In Fig. 5 of the version of this Article originally published, the final number on the $x$ axes of each panel was incorrectly written as 1.5 ; it should have read 7.5. This has now been corrected in all versions of the Article. 